International Journal of Modern Physics A

(C) World Scientific Publishing Company

\title{
QUANTUM SYSTEMS AND ALTERNATIVE UNITARY DESCRIPTIONS
}

\author{
G. MARMO \\ Dip. Scienze Fisiche and INFN Sez. di Napoli, Università Federico II , Compl. Univ. Monte \\ S.Angelo \\ Napoli, 80126, Italy * \\ A. SIMONI \\ Dip. Scienze Fisiche and INFN Sez. di Napoli, Università Federico II , Compl. Univ. Monte \\ S.Angelo \\ Napoli, 80126, Italy ${ }^{\dagger}$ \\ F. VENTRIGLIA \\ Dip. Scienze Fisiche and INFM Unità di Napoli, Università Federico II , Compl. Univ. Monte \\ S.Angelo \\ Napoli, 80126, Italy $\ddagger$ \\ Received (Day Month Year) \\ Revised (Day Month Year)
}

\begin{abstract}
Motivated by the existence of bi-Hamiltonian classical systems and the correspondence principle, in this paper we analyze the problem of finding Hermitian scalar products which turn a given flow on a Hilbert space into a unitary one. We show how different invariant Hermitian scalar products give rise to different descriptions of a quantum system in the Ehrenfest and Heisenberg picture.
\end{abstract}

Keywords: Quantum systems; completely integrable systems; symplectic dynamics.

\section{Introduction}

Classical bi-Hamiltonian systems have played a relevant role in the past decades for the study of completely integrable systems, both for finite and infinite number of degrees of freedom (see Ref. 1 and Ref. 2 for quantum systems).

Quantum systems admitting alternative commutation relations have been considered many times since the pioneering paper of E.P. Wigner ${ }^{3}$, see also Ref. 4, 5, 6 .

\footnotetext{
*marmo@na.infn.it

†simoni@na.infn.it

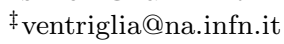


Quantum systems described by non Hermitian operators and possessing a real spectrum have been analyzed by several authors (e.g. Ref. 7, 8).

In a recent pape ${ }^{9}$ we have shown that these situations may be better tackled within the framework of the "inverse problem for quantum systems". From a mathematical point of view a similar problem was first discussed by Nagy 18 long ago.

To clearly formulate this problem let us briefly recall first the symplectic inverse problem for classical linear systems. Starting with a vector field

$$
\Gamma=\Gamma^{i} \frac{\partial}{\partial \xi^{i}}
$$

one searches far all possible Hamiltonian descriptions in terms of symplectic structures

$$
\omega=\omega_{j k} d \xi^{j} \wedge d \xi^{k}
$$

by solving for the equation $L_{\Gamma} \omega=0$. Every symplectic structure admits an "inverse", it is a bivector field, usually called a Poisson tensor, defined by $\Lambda^{i k} \omega_{k j}=\delta_{j}^{i}$. Thus, the inverse problem amounts to search for all decompositions of $\Gamma$ as the product

$$
\Gamma^{i}=\Lambda^{i k} \frac{\partial H}{\partial \xi^{k}}
$$

Where $\Lambda^{i k}$ is a skewsymmetric real, point dependent, matrix. If we deal with linear vector fields and quadratic Hamiltonians, say

$$
\Gamma^{i}=A_{k}^{i} \xi^{k} \quad, \quad H=\frac{1}{2} \xi^{k} H_{k j} \xi^{j},
$$

the inverse problem becomes a problem of linear algebra, i.e. searching for all decompositions of the dynamical matrix $A$ into the product of a non degenerate skew-symmetric matrix $\Lambda$ and a symmetric matrix $H$, in compact form $A=\Lambda \cdot H$. When $\Lambda$ is not required to be non-degenerate, we are dealing with the "inverse problem for Poisson dynamic" 10 We should remark that this problem is more interesting when we are considering non-linear situations and non constant rank of $\Lambda$, otherwise, by quotienting with respect to the kernel of $\Lambda$, we may go back to the symplectic situation.

Thus all possible decompositions of $A$, in the stated form, provide us with alternative Hamiltonian descriptions. In many physical situations, when dealing with completely integrable systems, we are interested in the existence of alternative decompositions once one has been already given, i.e. we search for alternative Hamiltonian descriptions for a given Hamiltonian system $\Gamma$. The alternative descriptions would characterize $\Gamma$ as a multi-Hamiltonian vector field. It is almost obvious that many alternative Hamiltonian descriptions will be generated by symmetries for $\Gamma$ which are not canonical transformations for $\Lambda$. However this way of generating new Hamiltonian descriptions will not exhaust the class of alternative ones. For 
instance 7 the two dimensional isotropic Harmonic Oscillator has different decompositions with either $H$ definite positive or with signature $(+,+,-,-)$, which arise from the following Hamiltonian descriptions:

$$
\begin{aligned}
& H_{0}=\frac{1}{2}\left(p_{1}^{2}+q_{1}^{2}+p_{2}^{2}+q_{2}^{2}\right) \\
& \omega_{0}=d p_{1} \wedge d q_{1}+d p_{2} \wedge d q_{2}
\end{aligned}
$$

and

$$
\begin{aligned}
& H=\frac{1}{2}\left(p_{1}^{2}+q_{1}^{2}-p_{2}^{2}-q_{2}^{2}\right) \\
& \omega=d p_{1} \wedge d q_{1}-d p_{2} \wedge d q_{2} .
\end{aligned}
$$

These factorizations cannot be related by any similarity transformation. In this setting, it has been shown 11 that when the critical point of the linear vector field $\Gamma$ is stable in the Liapunov sense, among the alternative Hamiltonian descriptions there is one which is positive definite.

The formulation of the inverse problem for quantum systems is now quite natural: given a vector field $\Gamma$ on some vector space $V$, we search for all Hermitian structures $h$ which are solution of the equation $L_{\Gamma} h=0$.

In our previous papers Ref. 7, 17, to avoid technicalities, we have carried on our study of the inverse problem within the framework of finite dimensional Hilbert spaces; in this paper we would like to consider the problem in the more relevant case of infinite dimensional Hilbert space.

This paper is organized as follows: in Sections 2, 3, 4, 5 the problem is specified, mathematical results are reviewed, some applications are given and the dependence of results on the fiducial metric $h_{0}$ is studied. In Section 6 we give some results on the existence of alternative invariant Hermitian scalar products; in Section 7 a way for finding invariant scalar products for Abelian groups is considered; in Section 8 we deal with the more general situation of related operators instead of similar ones; in Section 9 we apply our considerations to the Heisenberg group. Finally in Section 10 we describe quantum systems as Hamiltonian systems and discuss the consequences of the existence of alternative invariant scalar products, in Section 11 we draw some conclusions. Results which are available in the literature are referred here as Theorems, while our considerations are recast in Propositions and Corollaries.

\section{The Inverse Problem for Quantum Systems}

We consider a linear dynamical system $\Gamma$ on a complex vector space of states $\mathbb{H}$ carrying a Hilbert space structure $h_{0}$ (we define all scalar products to be linear in their second factor). Then $\Gamma$

$$
\Gamma: \mathbb{H} \rightarrow T \mathbb{H}=\mathbb{H} \times \mathbb{H} \quad, \quad \Gamma(\Psi)=\left(\Psi,-\frac{i}{\hbar} H \Psi\right)
$$

determines the differential equation

$$
\frac{d}{d t} \psi=-\frac{i}{\hbar} H \psi
$$


where $H$ is a linear operator on $\mathbb{H}$, but we do not require any Hermiticity properties with respect to $h_{0}$. Hereafter we put $\hbar=1$.

The inverse problem consists of finding which conditions have to be satisfied by $\Gamma$ for the existence of Hermitian scalar products invariant under the time evolution associated with $\Gamma$.

In finite dimensions, i.e. for a complex linear vector field on $\mathbb{C}^{n}$, we have $\mathbb{7}^{7}$ the following Proposition:

Proposition 1: A complex linear vector field $\Gamma(\Psi)=(\Psi,-i H \Psi)$ generates a flow $\phi_{t}: \mathbb{C}^{n} \rightarrow \mathbb{C}^{n}$ preserving some Hermitian scalar product $h$, i.e. $\phi_{t}^{*} h=h$, iff any one of the following equivalent conditions is satisfied:

1) $H=H^{\dagger}$, where the adjoint is taken with respect to the scalar product defined by $h$, i.e. $L_{\Gamma} h=0$;

2) $H$ is diagonalizable and has a real spectrum;

3) all the orbits $e^{-i H t} \Psi$ are bounded sets, for any initial condition $\Psi$.

Remark. Sometimes, properties stated in 2) are derived by requiring that the Hamiltonian is $\mathcal{P} \mathcal{T}$-symmetric (see for instance Ref. 12, 13).

The proof of this Proposition relies on the existence of a decomposition of $H$ into a nilpotent part and a semisimple part, commuting among themselves. Once we exponentiate this decomposition, the boundedness condition rules out the nilpotent part and requires that the semisimple part of $-i H$ has only imaginary eigenvalues. When going to infinite dimensions one may try to use a similar procedure, however the corresponding separation of $-i H$ holds true only for a special class of operators 14 Therefore we have to use a different approach.

When dealing with infinite dimensions, it is clear that, according to Weyl, 15 condition 3) will play a more convenient role because it is stated in terms of (what is going to become) the bounded operators $e^{-i H t}$ instead of the infinitesimal generator $-i H$ which, in the most physical situations, turns out to be unbounded and therefore would raise domain problems which make statements more cumbersome.

Therefore, within Weyl's ideology, it is better to deal with finite transformations (i.e. automorphisms of the state space) rather than infinitesimal transformations (i.e. endomorphisms, which, in general, create domain problems). This step, when starting with Eq. (8), may be achieved by using the Cayley map, i.e. by replacing $H$ with

$$
T=(H-i \mathbb{I})(H+i \mathbb{I})^{-1} .
$$

For a recent, authoritative analysis of this map see Kostant-Michor ${ }^{16}$ In this way we search for scalar products turning $T$ into a unitary operator and accordingly $H$ into a self-adjoint operator with a unitary flow $e^{-i H t}$.

Of course we could also decide to formulate the inverse problem in quantum mechanics directly in terms of one-parameter groups of automorphisms for the state space and to seek for all Hermitian products which turn the one-parameter group of transformations into a unitary one-parameter group. We shall therefore start with 
automorphisms of the state space instead of endomorphisms. To set the stage, we consider the vector space of states to be a Hilbert space, i.e. topology, completeness and bases are defined with respect to a chosen fiducial scalar product $h_{0}$. However this Hermitian structure need not be invariant under the transformations we are dealing with.

In the next Section we review few relevant results scattered in the existing literature, they were motivated by the search for stability criteria for infinite dimensional systems (compare the paragraph after formula Eq. (6) of the Introduction).

\section{Uniformly Bounded Operators}

Inspired by condition 3) of the above Proposition 1 we may consider an automorphism $T$ of a Hilbert space $\mathbb{H}$ with a Hermitian scalar product $h_{0}$ and construct the orbits

$$
\left\{T^{k} \Psi\right\} \quad ; \quad k \in\{0 . \pm 1, \pm 2, \ldots\}
$$

and require that all of them, with respect to the norm induced by $h_{0}$, are bounded sets for any value of $\Psi$. The use of the principle of uniform boundedness 27 shows that this is equivalent to require that $T$ is uniformly bounded.

We recall that the automorphism $T$ on $\mathbb{H}$ is said to be uniformly bounded if there exists an upper bound $c<\infty$ such that

$$
\left\|T^{k}\right\|<c ; \quad k \in\{0 . \pm 1, \pm 2, \ldots\} .
$$

Condition (11) is called Nagy criterion. For such an operator $T$ the following Theorem 18 holds:

Theorem 2: For a uniformly bounded operator $T$ there exists a bounded positive selfadjoint transformation $Q$ such that

$$
\frac{1}{c} \mathbb{I} \leq Q \leq c \mathbb{I}
$$

and $Q T Q^{-1}=U$ is unitary with respect to the fiducial $h_{0}$. This implies that $T=$ $Q^{-1} U Q$ is unitary with respect to

$$
h_{T}(X, Y):=h_{0}\left(Q^{2} X, Y\right) .
$$

Proof: (Sketch) The essential idea of the proof is to define the invariant scalar product $h_{T}(X, Y)$ as the limit, for $n$ going to infinity, of $h_{0}\left(T^{n} X, T^{n} Y\right)=: h_{n}(X, Y)$. This is the limit of a bounded sequence of complex numbers which does not exist in general, at least in the usual sense. Therefore a generalized concept of limit for bounded sequence, introduced by Banach and Mazur 19 , has to be used. This generalized limit (denoted as Lim) amounts to define the invariant scalar product $h_{T}$ as the transformed scalar product $h_{n}$ "at infinity" , where $T$ is interpreted as the generator of a $\mathbb{Z}$-action on $\mathbb{H}$. 
It is possible $\frac{18}{18}$ to use the same approach to deal with an $\mathbb{R}$-action instead of the $\mathbb{Z}$-action so that:

Theorem 3: When the one-parameter group of automorphisms $T(s)$ of linear transformations is uniformly bounded, that is $\|T(s)\|<c, s \in(-\infty, \infty)$, there exists a bounded selfadjoint transformation $Q$ such that $Q T(s) Q^{-1}=U(s)$ is a one-parameter group of unitary transformations. Clearly continuity properties with respect to $s$ are the same for both $T(s)$ and $U(s)$.

Remark. The iterated application of the uniformly bounded operator $T$ may be viewed as a discrete time evolution, which, according to Theorem 2, can be made unitary. Here we note that such a discrete time evolution may be fitted within a continuous differentiable time evolution. We have the following

Proposition 4: If $T$ is a uniformly bounded operator then there exists a bounded operator $A$, selfadjoint with respect to the invariant product $h_{T}$, such that $T^{n}=e^{i A n}$ for any $n \in \mathbb{Z}$.

Proof: From Theorem 2 we know that $T$ is $h_{T}$-unitary so that it can be written as

$$
T=\int_{0}^{2 \pi} e^{i \lambda} d E_{\lambda}^{T},
$$

where $E_{\lambda}^{T}$ is the uniquely defined spectral family for $T$ with the property $E_{0}^{T}=0$ and $E_{2 \pi}^{T}=\mathbb{I}$.

Now define $A=\int_{0}^{2 \pi} \lambda d E_{\lambda}^{T}$, so that $T^{n}=e^{i A n}$. The operator $A$ is of course $h_{T}$-selfadjoint, bounded and defined on the entire Hilbert space.

Of course, the one-dimensional unitary group $e^{i A t}$ is a continuous one-parameter group containing the discrete subgroup $\left\{T^{n}\right\}$ and moreover all orbits $e^{i A t} \Psi$, for any $\Psi \in \mathbb{H}$, are differentiable in $t$ and solve the Schroedinger equation for $A$. It is possible to go from one-parameter groups of transformations to finitely-manyparameters groups of transformations if they define an Abelian group. The following Theorem (see for instance Ref. 20) holds:

Theorem 5: A uniformly bounded action on $\mathbb{H}$ of an Abelian group $\mathcal{G}$, i.e.

$$
\|G\|<c \quad, \quad \forall G \in \mathcal{G}
$$

can be turned into a unitary action with the help of a bounded selfadjoint transformation $Q$, i.e. $Q G Q^{-1}$ are unitary transformations for any $G \in \mathcal{G}$.

The proof of this Theorem cannot use the idea of the generalized Banach limit, like in the previous more restricted cases, because no assumptions are made on the 
structure and the topology of $\mathcal{G}$. In fact, the proof relies on a very general result on the existence of fixed points for any continuous transformation of convex sets (Ref. 21, 22, see also Ref. 23).

For practical purposes, when Nagy condition is not easy to check, some equivalent conditions may be used (see Ref. 24, 25). It has been shown that Nagy condition Eq. (III) is equivalent to

$$
\begin{aligned}
& \sup _{|\lambda|>1}\left(|\lambda|^{2}-1\right) \int_{0}^{2 \pi}\left\|(T-\lambda)^{-1} u\right\|^{2} d \theta \leq C\|u\|^{2} \\
& \sup _{|\lambda|>1}\left(1-|\lambda|^{-2}\right) \int_{0}^{2 \pi}\left\|\left(T^{\dagger}-\lambda^{-1}\right)^{-1} u\right\|^{2} d \theta \leq C\|u\|^{2}
\end{aligned}
$$

where $\theta=\arg \lambda$. By using the Cayley transform a condition for the similarity of an operator $L$ to a selfadjoint one is recovered as:

$$
\begin{aligned}
& \sup _{\varepsilon>0} \varepsilon \int_{R}\left\|(L-\lambda)^{-1} u\right\|^{2} d k \leq C\|u\|^{2} \\
& \sup _{\varepsilon>0} \varepsilon \int_{R}\left\|\left(L^{\dagger}-\lambda\right)^{-1} u\right\|^{2} d k \leq C\|u\|^{2}
\end{aligned}
$$

where $\lambda=k+i \varepsilon$.

In Theorems $2,3,5$ the commutativity hypothesis is crucial! $\frac{26}{2 n}$ the non commutative case one needs necessarily some other assumptions, for instance that $\mathcal{G}$ is a (representation of a) compact group. Then the existence of the invariant Haar measure guarantees that $\mathcal{G}$ is similar to a unitary representation.

In Section 9, we discuss an application of Theorems 2 and 3 to the Heisenberg group, a very special case of non-commutativity.

In the following we will be concerned mainly with Theorems 2 and 3.

\section{Applications}

All the examples that follows are applications of Theorem 2 and refer to operators which are not normal. This is so because of the following corollary to Nagy's Theorem 2:

Corollary 6: A normal operator $T$ either is already unitary or it is not similar to any unitary operator. Equivalently: A normal operator $T$ is unitary if and only if it satisfies the Nagy condition.

Proof: When $T$ is normal the operators appearing in its polar decomposition, $T=V|T|$, commute, so that $|T|$ satisfies Nagy's condition Eq. (11) together with $T$. Then $|T|$ is both similar to a unitary operator and positive selfadjoint. This implies that the spectrum of $|T|$ reduces to 1 , so that $|T|=\mathbb{I}$ and $T=V$.

Example 1) As a simple example consider the group of translation on the line realized on $L_{2}(\mathbb{R})$ with a measure which is not translationally invariant, i.e.

$$
\left(T_{t} \Psi\right)(x):=\Psi(x+t), \Psi \in L_{2}(\mathbb{R}, \rho(x) d x),
$$


where $\rho(x)$ is any function $0<\alpha<\rho(x)<\beta<\infty$ and denote by $h_{\rho}$ the corresponding scalar product. If the limit $\lim _{x \rightarrow-\infty} \rho(x)$ exists, say $\lim _{x \rightarrow-\infty} \rho(x)=a$, then it is trivial to compute the Banach limit because it agrees with a limit in the usual sense. In fact by Lebesgue Theorem we have:

$$
\begin{aligned}
& \lim _{t \rightarrow \infty} \int_{\mathbb{R}} \Psi^{*}(x+t) \Phi(x+t) \rho(x) d x=\lim _{t \rightarrow \infty} \int_{\mathbb{R}} \Psi^{*}(x) \Phi(x) \rho(x-t) d x= \\
& \int_{\mathbb{R}} \lim _{t \rightarrow \infty} \Psi^{*}(x) \Phi(x) \rho(x-t) d x=a \int_{\mathbb{R}} \Psi^{*}(x) \Phi(x) d x=a h_{0}(\Psi, \Phi) .
\end{aligned}
$$

This shows that the Banach limit gives $h_{T}(\Psi, \Phi)=a h_{0}(\Psi, \Phi)$, i.e. it is a multiple of the standard translation invariant scalar product. Therefore

$$
h_{T}(\Psi, \Phi)=h_{\rho}\left(Q^{2} \Psi, \Phi\right)=h_{\rho}\left(\left(\sqrt{\frac{a}{\rho}}\right)^{2} \Psi, \Phi\right),
$$

that is $Q=\sqrt{\frac{a}{\rho}}$ and

$$
\left(U_{t} \Phi\right)(x)=\left(Q T_{t} Q^{-1} \Phi\right)(x)=\sqrt{\frac{\rho(x+t)}{\rho(x)}} \Phi(x+t)
$$

is unitary in $L_{2}(\mathbb{R}, \rho(x) d x)$.

Example 2) The following example deals with a non-diagonalizable uniformly bounded operator $T$ defined on $L_{2}(\mathbb{R}, d x)$ as

$$
(T \Psi)(x):=f(x) \Psi(-x)
$$

where $f(x)$ is a bounded function:

$$
0<\alpha \leq|f(x)| \leq \beta<\infty .
$$

In other words $T$ is the product of the parity operator $P$ times the multiplicative bounded operator $f$. Note that $T$ is non-normal as

$$
f P P f^{*}-P f^{*} f P=|f(x)|^{2}-|f(-x)|^{2}
$$

which is not zero for a generic $f$. Moreover this function $f$ has to be chosen in such a way that $T$ satisfies Nagy's condition. For this, taking into account that:

for $n>0$

$$
\left(T^{n} \Psi\right)(x)=\left(\prod_{k=1}^{n} f\left((-1)^{k+1} x\right)\right) \Psi\left((-1)^{n} x\right) \quad n>0
$$

while for $n<0$

$$
\left(T^{n} \Psi\right)(x)=\left(\prod_{k=1}^{-n} f^{-1}\left((-1)^{k} x\right)\right) \Psi\left((-1)^{n} x\right) \quad n<0
$$

the condition ||$T^{n}||<K$ implies the functional relation $|f(x) f(-x)|=1$, which admits the general solution :

$$
f(x)=\frac{\mu(x)}{\mu(-x)} e^{i \varphi(x)}
$$


where $\mu(x)$ is a real function such that: $0<\mu_{1} \leq \mu(x) \leq \mu_{2}<\infty$.

The Banach limit is readily evaluated and results

$$
\begin{aligned}
& h_{T}(\Phi, \Psi)=\operatorname{Lim}_{n \rightarrow \infty} h_{0}\left(T^{n} \Phi, T^{n} \Psi\right)= \\
& =\frac{1}{2} \int \Phi^{*}(x) \Psi(x)\left(1+\frac{\mu^{2}(-x)}{\mu^{2}(x)}\right) d x=h_{0}\left(Q^{2} \Phi, \Psi\right)
\end{aligned}
$$

where $Q^{2}=\frac{1}{2}\left(1+\frac{\mu^{2}(-x)}{\mu^{2}(x)}\right)$ is a bounded positive operator, as expected. The operator $U_{T}$, similar to $T$, which is unitary with respect to the standard scalar product $h_{0}$ is

$$
U_{T}=Q T Q^{-1}=e^{i \varphi(x)} P .
$$

$U_{T}$ has only continuous spectrum given by

$$
\lambda_{ \pm}= \pm e^{\frac{i}{2}\left(\varphi\left(x_{0}\right)+\varphi\left(-x_{0}\right)\right)} ; x_{0} \in \mathbb{R}
$$

with corresponding generalized eigenfunctions $\Psi_{ \pm}(x)$ :

$$
\Psi_{ \pm}(x)=e^{\frac{i}{2} \varphi\left(x_{0}\right)} \delta\left(x-x_{0}\right) \pm e^{\frac{i}{2} \varphi\left(-x_{0}\right)} \delta\left(x+x_{0}\right) .
$$

Example 3) The following example is similar to the previous one but parity is now replaced by a translation of a fixed amount $a$ :

$$
\left(T_{a} \Psi\right)(x):=f(x) \Psi(x+a)
$$

where $f(x)$ is a bounded function

$$
0<\alpha \leq|f(x)| \leq \beta<\infty \quad .
$$

Imposing $\left\|T^{n}\right\|<K$ one gets that $f(x)=g(x) e^{i \phi(x)}$ with $g(x)$ real and positive such that $g(x+a)=g(x)^{-1}$ and $\phi(x)$ arbitrary and real. Then as before one gets $Q^{2}=\frac{1}{2}\left(1+g^{2}(x)\right)$. The spectrum of $T_{a}$ is continuous, indeed the equation:

$$
\left(T_{a} \Psi\right)(x)=g(x) e^{i \phi(x)} \Psi(x+a)=\mu \Psi(x)
$$

can be solved in the form $\Psi(x)=\sqrt{g(x)} e^{i(\lambda x+\chi(x))}$; then

$$
\left(T_{a} \Psi\right)(x)=T_{a} \sqrt{g(x)} e^{i(\lambda x+\chi(x))}=\sqrt{g(x)} e^{i(\lambda x+\chi(x+a)+\phi(x))} e^{i \lambda a} .
$$

Therefore $\chi(x)$ must fulfill the functional relation $\chi(x+a)=\chi(x)+\phi(x)$; this relation determines $\chi$ on the entire line once it is arbitrarily chosen on $[0, a]$. The continuous spectrum is then the entire circle $\mu=e^{i \lambda a}$.

\section{Dependence of the Invariant Metric on the Choice of the Initial One}

In this Section we analyze to what extent the invariant metric $h_{T}$ changes by a change of the starting fiducial metric $h_{0}$ to a topologically equivalent one $h_{0}^{\prime}$. Any change of $h_{0}$ to $h_{0}^{\prime}$ is parameterized by a positive definite Hermitian operator $C$ by the relation

$$
h_{0}(x, y)=h_{0}^{\prime}(C x, y)
$$


and we get by Banach limits two invariant scalar products $h_{T}(x, y)$ and $h_{T}^{\prime}(x, y)$, which are related by a similar relation

$$
h_{T}(x, y)=h_{T}^{\prime}(R x, y) .
$$

Proposition 7: Consider the above $C$ and $R$ and define $A$ in the following way:

$$
\operatorname{Lim}_{n \rightarrow \infty} h_{T}^{\prime}\left(A_{n} x, T^{n} y\right)=: F(x, y)=h_{T}^{\prime}(A x, y)
$$

where

$$
A_{n}=\left[C, T^{n}\right] .
$$

Then $R=C+A$ and $[A, T]=-[C, T]$.

Proof: From the definition it is trivial to show that $\left\{A_{n}\right\}$ is a set of uniformly bounded operators; therefore it makes sense to compute the bilinear functional $F(x, y)$ and the operator $A$ such that $F(x, y)=h_{T}^{\prime}(A x, y)$ is well defined via Riesz Theorem. Then it requires only algebraic manipulations to show both of the following results: $R=C+A$ and $[A, T]=-[C, T]$.

This shows also that $[R, T]=0$, as it should, because any operator connecting two $T$-invariant Hermitian scalar product necessarily commutes with $T$. We will see this in the next Section.

\section{Alternative Invariant Hermitian Structures}

Starting with the automorphism $T$, we may investigate for the existence of alternative Hermitian structures invariant under the $\mathbb{Z}$-group action generated by $T$. Such a $T$ could than be said to be a bi-unitary map.

Assume therefore that $T$ is uniformly bounded. For the time being we assume in addition that $T$ is diagonalizable and multiplicity free (i.e. the commutant of $T$ is Abelian 27). Choose then a bounded transformation $S$ with bounded inverse such that

$$
T=S^{-1} U S
$$

where $U$ is unitary. Theorem 2 guarantees that at least one such $S$ exists. Then

$$
h^{S}(x, y):=h_{0}\left(S^{\dagger} S x, y\right)
$$

turns $T$ into a unitary operator. Because of the uniformly boundedness of $T$, we may also use the Banach limit

$$
\operatorname{Lim}_{n \rightarrow \infty} h_{0}\left(T^{n} x, T^{n} y\right)=: h_{T}(x, y)
$$

to define a new invariant Hermitian structure.

By using an $h_{0}$-orthonormal basis $\left\{\varphi_{k}\right\}$ of eigenvectors of $U$

$$
U \varphi_{k}=e^{i \lambda_{k}} \varphi_{k} \quad ; \quad h_{0}\left(\varphi_{k}, \varphi_{j}\right)=\delta_{k, j}
$$


we find by explicit computation of the Banach limit:

$$
h_{T}(x, y)=\sum_{k} h_{0}\left(S x, \varphi_{k}\right) h_{0}\left(\varphi_{k}, S y\right)\left\|S^{-1} \varphi_{k}\right\|^{2},
$$

while:

$$
h^{S}(x, y)=\sum_{k} h_{0}\left(S x, \varphi_{k}\right) h_{0}\left(\varphi_{k}, S y\right)
$$

We see that the invariant Banach scalar product $h_{T}$ is obtained from $h^{S}$ scaling each $\varphi_{k}$ by a factor $\left\|S^{-1} \varphi_{k}\right\|$. Notice that any bounded sequence of positive numbers can be used in the same way to scale $\varphi_{k}$ to obtain alternative invariant scalar products. Thus the sequence $\{1,1,1 \ldots\}$ corresponds to $h^{S}(x, y)$ while $\left\{\left\|S^{-1} \varphi_{1}\right\|,\left\|S^{-1} \varphi_{2}\right\|, \ldots\right\}$ to $h_{T}(x, y)$.

If the eigenvalues of $U$ are not multiplicity free we need another index $l$ to label eigenvectors; then Eq. (44) becomes

$$
h_{T}(x, y)=\sum_{k, l, n} h_{0}\left(S x, \varphi_{k l}\right) h_{0}\left(\varphi_{k n}, S y\right) h_{0}\left(S^{-1} \varphi_{k l}, S^{-1} \varphi_{k n}\right) .
$$

As in the multiplicity free case one obtains invariant scalar products replacing $h_{0}\left(S^{-1} \varphi_{k l}, S^{-1} \varphi_{k n}\right)$ with any bounded sequence of positive matrices.

What we learn from this example is that we can look for alternative Hermitian structures on each eigenspace of $U$ and then combine them with arbitrary positive coupling coefficients provided that bounded vectors remain bounded with respect to the newly defined products ( boundedness of the sequence). In this respect see our previous paper ${ }^{9}$ where similar considerations came out within the finite dimensional situation.

In particular this procedure shows that we may start with $h_{0}$ already invariant and construct $S$ out of constants of the motion for $T$, in this way we would obtain alternative descriptions whenever the sequence $\left\|S^{-1} \varphi_{k}\right\|$ is appropriate. For instance in a central force problem $2+\sin \left(J^{2}\right)$, in a basis for the angular momentum, would give $2+\sin j(j+1)$ and $1 /[2+\sin j(j+1)]$ would be an appropriate sequence.

Remark From what we said, it is clear that instead of a once-for-all chosen sequence of bounded positive numbers (for instance like $\{1,1,1 \ldots\}$ and $\left\{\left\|S^{-1} \varphi_{1}\right\|,\left\|S^{-1} \varphi_{2}\right\|, \ldots\right\}$ ) we could use a "point-dependent" sequence so that the Hermitian metric we define will be dependent on the point and therefore the "energy function" we associate with linear transformations will not be quadratic anymore. ${ }^{4}$ In this way, vector fields are still linear and therefore compatible with the dynamical linear superposition rule for state vectors, however the associated Hamiltonian functions (infinitesimal generators) are no more homogeneous of degree two. One is getting a kind of "non-linearity", one should compare this situation with the one proposed by Weinberg! 28

Having learned from the diagonalizable case, we can obtain a class of invariant scalar products dropping this assumption. Suppose again $T$ uniformly bounded. 
Then

$$
T=Q^{-1} U Q
$$

and

$$
h_{T}(x, y)=h_{0}\left(Q^{2} x, y\right)
$$

Consider the spectral decomposition of $U$ :

$$
U=\int_{0}^{2 \pi} e^{i \lambda} d E_{\lambda}^{U}
$$

Choose now any positive bounded function $\varphi$ on $[0,2 \pi]$ and define, in analogy with the diagonalizable case, the scalar product:

$$
h_{\varphi}(x, y)=\int_{0}^{2 \pi} \varphi(\lambda) h_{0}\left(Q x, d E_{\lambda}^{U} Q y\right)
$$

One checks easily that $h_{\varphi}(x, y)$ is also invariant and that $\varphi=1$ corresponds to the Nagy product $h_{T}(x, y)$.

Via Riesz Theorem, we may write $h_{\varphi}(x, y)=h_{T}\left(C_{\varphi} x, y\right)$ and solve for $C_{\varphi}$. To this aim we define $B$ as

$$
B=\int_{0}^{2 \pi} \varphi(\lambda) d E_{\lambda}^{U}
$$

to get

$$
\begin{aligned}
& h_{\varphi}(x, y)=\int_{0}^{2 \pi} \varphi(\lambda) h_{0}\left(Q x, d E_{\lambda}^{U} Q y\right)=h_{0}(Q x, B Q y)= \\
& =h_{0}\left(Q x, Q Q^{-1} B Q y\right)=h_{T}\left(x, Q^{-1} B Q y\right) .
\end{aligned}
$$

This formula furnishes $C_{\varphi}=Q^{-1} B Q$.

It is not hard to show that $T$ commutes with $C_{\varphi}$, as

$$
h_{\varphi}(T x, T y)=h_{\varphi}(x, y)=h_{T}\left(C_{\varphi} x, y\right)=h_{T}\left(C_{\varphi} T x, T y\right)=h_{T}\left(T C_{\varphi} x, T y\right)
$$

so that $\left[T, C_{\varphi}\right]=0$ follows.

We conclude this Section by noting that the class of invariant scalar product compatible with the starting one is parameterized by the definite positive elements in the commutant of $T$, and in particular it is empty only when $T$ is not uniformly bounded. 


\section{Invariant Hermitian Structures for Commuting Uniformly Bounded Operators}

The method of finding an invariant scalar product for an automorphism $T$ via a limiting procedure "at infinity" applies also in the case of many uniformly bounded commuting automorphisms.

We analyze first the case of two uniformly bounded commuting operators $T_{1}$, $T_{2}$. They generate an action of the Abelian group $\mathbb{Z} \times \mathbb{Z}$ by uniformly bounded operators; Theorem 5 guarantees the existence of an invariant Hermitian scalar product. We show how to compute one of them.

One first compute $h_{T_{1}}$ as a Banach limit; this is by construction invariant under $T_{1}$ but in general not under $T_{2}$. As second step one defines:

$$
h_{12}(x, y)=\operatorname{Lim}_{n \rightarrow \infty} h_{T_{1}}\left(T_{2}^{n} x, T_{2}^{n} y\right) .
$$

Proposition 8: Consider two uniformly bounded commuting operators $T_{1}, T_{2}$. Then

(i) $h_{1,2}$, defined in Eq. 54), is invariant under the action of the entire $\mathbb{Z} \times \mathbb{Z}$.

(ii) If $T_{1}$ is multiplicity free, $h_{T_{1}}$ is already invariant under the action of the entire $\mathbb{Z} \times \mathbb{Z}$

Proof: The proof of (i) is trivial. To prove (ii) consider $C_{1}$ defined through

$$
h_{T_{1}}(x, y)=: h_{12}\left(C_{1} x, y\right) .
$$

As shown before $C_{1}$ commutes with $T_{1}$, but $T_{1}$ is multiplicity free and therefore its commutant is Abelian. So $T_{2}$ must commute with $C_{1}$ and the result follows at once from Eq. (55).

Remark: These results may obviously be extended to any uniformly bounded action of the product of a finite number of groups $\mathbb{Z}$ and/or $\mathbb{R}$.

Corollary 9: Suppose that, in a uniformly bounded action of an Abelian group, a particular operator $\widetilde{T}$ is multiplicity free. Then the corresponding Nagy product $h_{\widetilde{T}}$ is invariant for the entire action.

Proof: The result follows readily extending the argument of the proof of the above point (ii) to the general case of Theorem 5 .

\section{Relating Two Uniformly Bounded Operators via Banach Limits}

The uniform boundedness condition is necessary and sufficient for an operator $T$ to be similar to a unitary one $U$ as stated in Theorem 2, then $T$ and $U$ have the same spectrum. The $A$-relatedness property is a condition weaker than similarity that has been utilized to discuss in more general terms operators and relations between 
their spectra, see for instance Ref. 17 . We recall that two operators $T_{1}$ and $T_{2}$ are said to be $A$-related if

$$
T_{1} A=A T_{2}
$$

then the operator $A$ is called an intertwining operator.

In this Section we discuss the case of two not necessarily commuting operators $T_{1}$ and $T_{2}$ both uniformly bounded and ask for conditions under which there exists a nontrivial operator $A$ intertwining them. We require also that $A$ should be bounded.

For this discussion consider the bilinear functional $\mathcal{F}(x, y)$ defined as follows:

$$
\mathcal{F}(x, y):=\operatorname{Lim}_{n \rightarrow \infty} h_{0}\left(T_{2}^{n} x, T_{1}^{n} y\right)
$$

this limit is well defined for all $x, y \in \mathbb{H}$ and a simple computation shows that $|\mathcal{F}(x, y)| \leq K\|x\|\|y\|$.

Using Riesz Theorem we define three bounded operators $A_{0}, A_{1}, A_{2}$ via:

$$
\mathcal{F}(x, y)=h_{0}\left(A_{0} x, y\right)=h_{T_{1}}\left(A_{1} x, y\right)=h_{T_{2}}\left(A_{2} x, y\right) .
$$

The following proposition holds:

Proposition 10: With $\mathcal{F}, A_{0}, A_{1}, A_{2}$ defined as above the relations 1-4 hold:

(1) $A_{0}=Q_{1}^{2} A_{1}=Q_{2}^{2} A_{2}$;

(2) $A_{0} T_{2}=\left(T_{1}^{\dagger}\right)^{-1} A_{0}$, the adjoint is with respect to the $h_{0}$ scalar product;

(3) $A_{1} T_{2}=\left(T_{1}^{\dagger}\right)^{-1} A_{1}$, the adjoint is with respect to the $h_{T_{1}}$ scalar product;

(4) $A_{2} T_{2}=\left(T_{1}^{\dagger}\right)^{-1} A_{2}$, the adjoint is with respect to the $h_{T_{2}}$ scalar product.

Proof: These relations follow at once from the definitions of the $A$ 's operators and the invariance property of $\mathcal{F}: \mathcal{F}\left(T_{2} x, T_{1} y\right)=\mathcal{F}(x, y)$.

Remark. Relation (3) shows that $T_{1}$ and $T_{2}$ are $A_{1}$-related when $\mathcal{F} \neq 0$, because in this case it results that $\left(T_{1}^{\dagger}\right)^{-1}=T_{1}$.

We therefore are led to examine some conditions which guarantees that $\mathcal{F} \neq 0$. Consider the simple case of $T_{1}$ and $T_{2}$ both diagonalizable and multiplicity free. Recalling that in our hypothesis on $T_{1}$ and $T_{2}$ we have:

$$
T_{1}=Q_{1}^{-1} U_{1} Q_{1} \quad, \quad T_{2}=Q_{2}^{-1} U_{2} Q_{2},
$$

it is easy, by using two suitable orthonormal basis, to obtain the following expression for $\mathcal{F}$ :

$$
\mathcal{F}(x, y)=\sum_{k, q} \delta_{\lambda_{k}, \mu_{q}} h_{0}\left(x, Q_{2} \psi_{q}\right) h_{0}\left(Q_{2}^{-1} \psi_{q}, Q_{1}^{-1} \varphi_{k}\right) h_{0}\left(Q_{1} \varphi_{k}, y\right)
$$

where $U_{1} \varphi_{k}=\lambda_{k} \varphi_{k}, U_{2} \psi_{q}=\mu_{q} \psi_{q}$ and $h_{0}\left(\varphi_{k}, \varphi_{j}\right)=\delta_{k, j}, h_{0}\left(\psi_{k}, \psi_{j}\right)=\delta_{k, j}$.

We have shown therefore that

Proposition 11: If $T_{1}$ and $T_{2}$ are uniformly bounded, diagonalizable and multiplicity free operators then $\mathcal{F} \neq 0$ if and only if they have at least one common eigenvalue and $h_{0}\left(Q_{2}^{-1} \psi_{q}, Q_{1}^{-1} \varphi_{k}\right) \neq 0$. 
We note also that from Eq. (60) it is easy to obtain an explicit expression for the intertwining operators $A$ 's. For instance:

$$
A_{0}=\sum_{k, q} \delta_{\lambda_{k}, \mu_{q}} h_{0}\left(Q_{1}^{-1} \varphi_{k}, Q_{2}^{-1} \psi_{q}\right) h_{0}\left(Q_{2} \psi_{q}, \cdot\right) Q_{1} \varphi_{k}
$$

An argument similar to the one used after Eq. (44) shows that one can replace in Eq. (61) $h_{0}\left(Q_{1}^{-1} \varphi_{k}, Q_{2}^{-1} \psi_{q}\right)$ with any bounded sequence of numbers, obtaining in this way other intertwining operators.

One can use Eq. (61) even in cases involving continuous spectra. For instance in $L_{2}(\mathbb{R}, d x)$ consider $\left(T_{1} \Psi\right)(x)=e^{i x} \Psi(x)$ and $\left(T_{2} \Psi\right)(x)=\Psi(x+a), a \in \mathbb{R}$; then, when the sequence $h\left(Q_{1}^{-1} \varphi_{k}, Q_{2}^{-1} \psi_{q}\right)$ is replaced by a constant sequence, Eq. (61) leads to the Fourier transform operator.

\section{Invariant Hermitian Structures for Realizations of the Heisenberg Group}

Elsewhere we have shown 29 how alternative symplectic structures, on a finite dimensional real symplectic vector space $V$, give rise to alternative Weyl systems, i.e. alternative projective unitary representations of the Abelian vector group $V$.

In this Section we show that it is possible to find invariant Hermitian structures for any realization of the Heisenberg group in terms of uniformly bounded operators.

We consider operators $T_{1}, T_{2}, T_{3}$ uniformly bounded and obeying the following commutation relations

$$
T_{1} T_{3} T_{1}^{-1} T_{3}^{-1}=\mathbb{I}=T_{2} T_{3} T_{2}^{-1} T_{3}^{-1}
$$

and

$$
T_{1} T_{2} T_{1}^{-1} T_{2}^{-1}=T_{3}
$$

Proposition 12: For $T_{1}, T_{2}, T_{3}$ satisfying the stated conditions it is possible to find a Hermitian structure that converts them into unitary operators.

Proof: There is a scalar product, say $h_{13}$, that makes $T_{1}$ and $T_{3}$ unitary since they commute and are uniformly bounded; in this scalar product $T_{2}$ will not be unitary in general. Consider therefore

$$
h(x, y):=\operatorname{Lim}_{n \rightarrow \infty} h_{13}\left(T_{2}^{n} x, T_{2}^{n} y\right) .
$$

This scalar product $h$ makes $T_{2}$ unitary and one checks easily that it leaves unitary $T_{1}$ and $T_{3}$ as well. In fact

$$
\begin{aligned}
h\left(T_{1} x, T_{1} y\right) & =\operatorname{Lim}_{n \rightarrow \infty} h_{13}\left(T_{2}^{n} T_{1} x, T_{2}^{n} T_{1} y\right)= \\
& =\operatorname{Lim}_{n \rightarrow \infty} h_{13}\left(T_{1} T_{3}^{n} T_{2}^{n} x, T_{1} T_{3}^{n} T_{2}^{n} y\right)=h(x, y)
\end{aligned}
$$

and the same for $T_{3}$.

In the future we will show the use of this proposition, to compare alternative Weyl systems when they are considered on the same Hilbert space. 


\section{Quantum Systems as Hamiltonian Systems}

Let us recall that for a complex Hilbert space $\mathbb{H}$ with Hermitian structure $h$ it is possible to define a symplectic structure by setting

$$
\omega_{h}(x, y):=\mathfrak{I m} h(x, y) \quad .
$$

Given a symplectic vector space $(V, \omega)$ we may define a Poisson Bracket on $V^{*}$ by defining it first on linear functions and then by using the Leibnitz rule on all differentiable functions $\mathcal{F}\left(V^{*}\right)$. On $\operatorname{Lin}\left(V^{*}, \mathbb{C}\right) \subset \mathcal{F}\left(V^{*}\right)$ we set

$$
\left\{v_{1}, v_{2}\right\}=\omega\left(v_{1}, v_{2}\right)
$$

where on the left hand side $v_{1}, v_{2} \in \mathcal{F}\left(V^{*}\right)$ and on the right $v_{1}, v_{2} \in V$.

More directly, on any totally reflexive space 30 , it is possible to define a nondegenerate Poisson Bracket if the space is strongly symplectic. ${ }^{31}$ Indeed introducing differentials of functions

$$
d f: V^{*} \rightarrow V^{*} \times V^{* *} \equiv V^{*} \times V
$$

in intrinsic form at each point $\alpha \in V^{*}$, we define

$$
\{f, g\}(\alpha):=\omega(d f(\alpha), d g(\alpha)) .
$$

In our setting $V$ is a Hilbert space and therefore there is a non intrinsic isomorphism between $V$ and $V^{*}$ so that we have a Poisson Bracket defined also on $\mathcal{F}(V)$. It follows easily that a complex unitary linear transformation $U$ on $\mathbb{H}$ is symplectic. A densely defined complex linear operator $\Gamma$ is a Hamiltonian vector field iff $H$ is Hermitian. The Hamiltonian function associated with $\Gamma$ is given by the formula

$$
f_{H}(\psi)=\frac{1}{2} h(H \psi, \psi) \quad \psi \in D(\Gamma) .
$$

Thus, we associate an Hamiltonian function (infinitesimal generating function) with any vector field which preserves the Hermitian structure $h$.

Disregarding domain problems, for the moment, it is easy to show that if $A, B, \ldots .$. are Hermitian operators, the associated Hamiltonian functions, say $f_{A}, f_{B}, \ldots$, satisfy the Poisson Bracket relations

$$
\left\{f_{A}, f_{B}\right\}(\psi)=f_{i[A, B]}(\psi) .
$$

It is now possible to investigate the consequence of the existence of alternative invariant Hermitian structures. With the notation of Theorem 2, we have a new Poisson Bracket defined by

$$
\left\{v_{1}, v_{2}\right\}_{T}=i \omega_{h}\left(Q^{2} v_{1}, v_{2}\right)
$$

These new metrics will associate alternative quadratic functions with every operator $A$. All of the induced Poisson Bracket on quadratic functions will be pairwise compatible in the sense of bi-Hamiltonian systems. In the Ehrenfest description of quantum dynamics, we have

$$
i \hbar \frac{d}{d t} f_{A}=\left\{f_{H}, f_{A}\right\}
$$


and therefore the same vector field will be given different Hamiltonian descriptions, with $f_{H}$ and the Poisson Bracket depending on the chosen invariant metric.

The relation $h\left(v_{1}, v_{2}\right)=h^{\prime}\left(R v_{1}, v_{2}\right)$, between two invariant Hermitian forms, suggests the correspondence between operators

$$
N: A \mapsto R A
$$

According to the Ref. 32, we may define a new associative product on the space of operators

$$
A \circ{ }_{N} B=N(A) B+A N(B)-N(A B)
$$

which gives $A \circ_{N} B=A R B$. Any time that $R$ is a constant of the motion for $H$, as in this case, we obtain a new alternative associative product on the space of operators which makes $H$ into an inner derivation.

In conclusion, we have shown how alternative Hamiltonian descriptions for the Schroedinger equation give rise to alternative description in the Ehrenfest picture and the Heisenberg picture.

\section{Conclusions}

In this paper we have addressed the problem of alternative quantum Hamiltonian descriptions of the same vector field on the space of quantum states. In this way we have avoided dealing with the ambiguity of quantization procedures for classical bi-Hamiltonian systems.

We have briefly addressed the question of the alternative descriptions at the level of the Ehrenfest picture and Heisenberg picture. By using this results, in the future we shall consider the quantum-classical transition to show how these alternative description at the quantum level will reproduce known alternative Hamiltonian descriptions for the corresponding classical systems.

\section{References}

1. F.Magri, J.Math.Phys. 19, 1156 (1978); F.Magri, Lect.Notes in Phys. 120, 233 (1980); B.Fuchssteiner, Progr.Theor.Phys. 68, 1082 (1982); S.De Filippo, G.Marmo and G.Vilasi, Nuovo Cimento B 83, 97 (1984).

2. A.M. Bloch, Phys. Lett. A 116, 353 (1986); A.M. Bloch, Trans. Am. Math. Soc. 302, 787 (1987).

3. E.P.Wigner, Phys. Rev. 77711 (1950).

4. B.A. Dubrovin, G. Marmo and A. Simoni: Mod. Phys. Lett.A 5, 1299 (1990).

5. V.I.Man'ko, G.Marmo, E.C.G.Sudarshan and F.Zaccaria, Int.J.Mod.Phys. 11 B, 1281 (1997).

6. J.Beckers, N.Debergh, J.Carinena and G.Marmo, Mod.Phys.Lett. A 16, 91 (2001); R. López-Peña, V. I. Manko, and G. Marmo, Phys. Rev. A 56, 1126 (1997).

7. F.Ventriglia, Mod. Phys.Lett. A 17,1589 (2002).

8. S.Albeverio, S-M.Fei and P.Kurasov, Lett. Math. Phys. 59, 227 (2002).

9. G.Marmo,G. Morandi, A.Simoni and F.Ventriglia, J.Phys. A: Math.Gen. 35, 8393 (2002). 
10. J.F.Carinena, L.A.Ibort, G.Marmo, A.Stern, Phys. Rep., 263, 153 (1995).

11. M.Giordano, G.Marmo and C.Rubano, Inverse Problems , 9, 443 (1993).

12. C.M.Bender and S.Boettcher, Phys. Rev. Lett. 80, 5243 (1998); C.M.Bender, Phys. Rep. 315, 27 (1999); C.M.Bender,G.V.Dunne, P.N. Meisinger and M.Simsek, Phys. Lett. A 281, 311 (2001); M.Znojil and M.Tatar, J.Phys. A: Math.Gen. 34, 1793 (2001); Z.Ahmed, Phys.Lett.A 290, 19 (2001); F.Cannata, G.Junker and J.Trost, Phys.Lett. A 245, 219 (1998); F.Cannata,M.Jofte, R. Roychoudbury and P.Roy, Phys.Lett. A 281, 305 (2001).

13. A. Mostafazadeh, J.Math.Phys. 43, 205 (2002); A. Mostafazadeh, J.Math.Phys. 43, $2814(2002)$

14. N. Dunford, J.T. Schwartz :Linear Operators; Part III, Wiley-Interscience, N.Y. (1971).

15. H.Weyl Theory of groups and quantum mechanics, Dover, N.Y. (1931).

16. B.Kostant,P.W. Michor, in The orbit method in geometry and physics: In honor of A.A.Kirillov, Eds.Duval, Guieu, Ovcienko, Progress in Mathematics 213, Birkauser Verlag (2003), 259-296.

17. G.Marmo, A.Simoni and F.Ventriglia, Rep. Math. Phys., 51, 275 (2003).

18. Bĕla de Sz. Nagy, On uniformly bounded linear transformations in Hilbert space, Acta Sci. Math., (Szeged, Hung.), 11, n.3, 152-157 (1945).

19. S.Banach, Theorie des operations lineaires, Chelsea Publishing Company, N.Y. (1955).

20. Ju. L. Daleckiĩ and M. G. Kreĩn, Stability of Solutions of Differential Equations in Banach Space, Translations of Mathematical Monographs, 43 Am. Math. Soc., Providence, Rhode Island, (1974).

21. A.Tychonoff, Ein Fixpunktsatz, Math. Ann., 111, 767-776 (1935).

22. A.Markov, Quelques Theoremes sur les ensembles abèliens, Comptes Rendus (Doklady) de l'Academie des Sciences de l'URSS, Vol. 1 (X) n. 8 (85), 311-313 (1936).

23. Y.Benyamini and J.Lindenstrauss, Geometric Nonlinear Functional Analysis, vol. 1, Am. Math. Soc., Providence Rhode Island, (1991).

24. S.N. Naboko, Funktsional. Anal. i Prilozhen (Functional Anal. Appl.), 18,n. 1, 16-27, (1984).

25. J.A. Van Casteren, Pacific J. Math., 104, 241 (1983).

26. G.Pisier,Similarity Problems and Completely Bounded Maps,2nd expanded ed., Springer-Verlag, Berlin (2001).

27. M.Reed and B.Simon, Functional Analysis I; Academic Press, N.Y.(1972).

28. S.Weinberg, Ann. of Phys. (N.Y), 194336 (1989).

29. G.Marmo, A.Simoni and F.Ventriglia, Rep. Math. Phys. 48, 149 (2001).

30. E.Nelson, Tensor Analysis, Princeton University Press, Princeton, (1967).

31. R. Abraham and J.E. Marsden, Foundations of Mechanics, 2nd ed., Addison-Wesley, Reading (1978).

32. G.Marmo, A.Simoni and F.Ventriglia, Rendiconti del Circolo Matematico di Palermo (suppl.) 69, 19 (2002). 